\title{
Zero-free neighborhoods around the unit circle for Kac polynomials
}

\author{
Gerardo Barrera' ${ }^{1}$ Paulo Manrique ${ }^{2}$
}

Accepted: 22 March 2021 / Published online: 7 August 2021

(c) The Author(s) 2021

\begin{abstract}
In this paper, we study how the roots of the Kac polynomials $W_{n}(z)=\sum_{k=0}^{n-1} \xi_{k} z^{k}$ concentrate around the unit circle when the coefficients of $W_{n}$ are independent and identically distributed nondegenerate real random variables. It is well known that the roots of a Kac polynomial concentrate around the unit circle as $n \rightarrow \infty$ if and only if $\mathbb{E}\left[\log \left(1+\left|\xi_{0}\right|\right)\right]<\infty$. Under the condition $\mathbb{E}\left[\xi_{0}^{2}\right]<\infty$, we show that there exists an annulus of width $\mathrm{O}\left(n^{-2}(\log n)^{-3}\right)$ around the unit circle which is free of roots with probability $1-\mathrm{O}\left((\log n)^{-1 / 2}\right)$. The proof relies on small ball probability inequalities and the least common denominator used in [17].
\end{abstract}

Keywords Locally sub-Gaussian random variables · Salem-Zygmund type inequalities · Small ball probability $\cdot$ Zeros of random polynomials

Mathematics Subject Classification Primary 60G99 - 12D10; Secondary $\cdot$ 11CXX $\cdot 30 \mathrm{C} 15$

\section{Introduction}

The $z$-transform (a particular case is the discrete Fourier transform) is an important tool in signal analysis and speech recognition. In this context, the study of the zeros of $z$-transforms provides useful information on a signal. The existence of a region free of zeros around the unit circle is important to the proper working of the $z$-transform. For further details, see Chapter 3 in [4]. Roughly speaking, in this paper we find a region free of zeros around the unit circle for Kac polynomials, which are closely related to the discrete Fourier transform.

G. Barrera was supported by CIMAT and PIMS. P. Manrique was supported by Cátedras-CONACyT, México.

$\bowtie$ Gerardo Barrera

gerardo.barreravargas@ helsinki.fi

Paulo Manrique

pmanriquem@ipn.mx

1 Department of Mathematics and Statistics, University of Helsinki, P.O. Box 68, 00014 Helsingin Yliopisto, Finland

2 General Coordination of Institutional Organization and Information, National Polytechnic Institute, 07738 Mexico City, Mexico 
Let $n \in \mathbb{N}$ and let $\xi_{0}, \ldots, \xi_{n-1}$ be independent and identically distributed (iid for short) nondegenerate real random variables (rvs for short) defined in the probability space $(\Omega, \mathcal{F}, \mathbb{P})$. Denote by $\mathbb{E}$ the expectation with respect to the measure $\mathbb{P}$. The Kac polynomial $W_{n}$ is defined as the random polynomial of degree $n-1$ given by

$$
W_{n}(z)=\sum_{j=0}^{n-1} \xi_{j} z^{j}, \quad z \in \mathbb{C} .
$$

In the sequel, we introduce the basic notation and terminology that will be used throughout this paper. For any $z \in \mathbb{C}$, let $|z|$ denote the modulus of $z$ and $\arg (z)$ the argument of $z$. Choose $a, b \in \mathbb{R}$ such that $a \leq b$. Let $R_{n}(a, b)$ denote the number of roots of $W_{n}$ in the annulus $\{z \in \mathbb{C}: a \leq|z| \leq b\}$ and, for any $\alpha, \beta \in[-\pi, \pi]$ such that $\alpha \leq \beta$, let $S_{n}(\alpha, \beta)$ denote the number of roots in $\{z \in \mathbb{C}: \alpha \leq \arg (z) \leq \beta\}$.

Shparo and Shur proved in [20] that under general conditions on the random coefficients (rcs for short), the roots of $W_{n}$ concentrate around the unit circle with asymptotically uniform distribution in the argument as $n$ increases. Moreover, Ibragimov and Zaporozhets showed in [7] that the rcs of $W_{n}$ are nondegenerate satisfying $\mathbb{E}\left[\log \left(1+\left|\xi_{0}\right|\right)\right]<\infty$ if and only if its roots are asymptotically concentrated near the unit circle. Later, Kabluchko and Zaporozhets provided in [9] a wide description of the localization of the roots for different conditions on the rcs. We point out that the localization of the roots of Kac polynomials determine the poor efficiency of some algorithms for speech recognition and signal processing applications; see [5] for further details.

Ibragimov and Zaporozhets proved in [7] that

$$
\mathbb{P}\left(\lim _{n \rightarrow \infty} \frac{1}{n} R_{n}(1-\delta, 1+\delta)=1\right)=1 \quad \text { holds for any } \delta \in(0,1)
$$

if and only if $\mathbb{E}\left[\log \left(1+\left|\xi_{0}\right|\right)\right]<\infty$. They also proved that for any distribution $\xi_{0}$ and $\alpha, \beta \in(-\pi, \pi)$ the following holds:

$$
\mathbb{P}\left(\lim _{n \rightarrow \infty} \frac{1}{n} S_{n}(\alpha, \beta)=\frac{\beta-\alpha}{2 \pi}\right)=1 .
$$

Shepp and Vanderbei studied in [19] the case of iid standard Gaussian coefficients and showed that

$$
\lim _{n \rightarrow \infty} \frac{1}{n} \mathbb{E}\left[R_{n}\left(e^{-\delta / n}, e^{\delta / n}\right)\right]=\frac{1+e^{-2 \delta}}{1-e^{-2 \delta}}-\frac{1}{\delta} \quad \text { for any } \delta>0 .
$$

Later, Ibragimov and Zeitouni, in [8], extended (1) to the case of iid coefficients, whose common distribution belongs to the domain of attraction of an $\alpha$-stable law:

$$
\lim _{n \rightarrow \infty} \frac{1}{n} \mathbb{E}\left[R_{n}\left(e^{-\delta / n}, e^{\delta / n}\right)\right]=\frac{1+e^{-\alpha \delta}}{1-e^{-\alpha \delta}}-\frac{2}{\alpha \delta} \quad \text { for any } \delta>0 .
$$

Note that for any $\delta>0$, as $\alpha \rightarrow 0^{+}$we have $\frac{1+e^{-\alpha \delta}}{1-e^{-\alpha \delta}}-\frac{2}{\alpha \delta} \rightarrow 0$. Then (2) may tend to zero as $n \rightarrow \infty$ when $\xi_{0}$ has a slowly varying tail distribution. In fact, Götze and Zaporozhets showed in [6] that if $\left|\xi_{0}\right|$ has a slowly varying tail distribution, then

$$
\lim _{n \rightarrow \infty} \mathbb{P}\left(R_{n}\left(e^{-\delta / n}, e^{\delta / n}\right)=0\right)=1 \quad \text { for any } \delta>0,
$$

i.e., the roots of a Kac polynomial with iid res with a slowly varying tail distribution hit the unit circle with almost zero probability. 
In the case that $W_{n}$ has iid rcs whose common distribution belongs to the domain of attraction of an $\alpha$-stable law, limit (2) yields that, for $\delta>0, W_{n}$ has at least one root in the annulus $R_{\delta, n}:=\left\{z \in \mathbb{C}: e^{-\delta / n} \leq|z| \leq e^{\delta / n}\right\}$ with positive probability for all large $n$ and

$$
\mathbb{P}\left(R_{n}\left(e^{-\delta / n}, e^{\delta / n}\right)=n\right) \leq \frac{1+e^{-\alpha \delta}}{1-e^{-\alpha \delta}}-\frac{2}{\alpha \delta}+\mathrm{o}(1) .
$$

Therefore, a remarkable question is to determine if there exists an annulus inside of $R_{\delta, n}$ such that $W_{n}$ has at least one root on it or not. The existence of roots pretty close to the unit circle is an important aspect in the analysis of signals. This helps us to understand the contribution of the phase information of a signal. We refer to [5] for further details.

Shepp and Vanderbei conjectured in [19] that, with high probability, the nearest root of $W_{n}$ to the unit circle is at a distance of order $\mathrm{O}\left(n^{-2}\right)$. Later, Konyagin and Schlag showed in [13] that the last conjecture holds true when the rcs have standard Gaussian or Rademacher (uniform distribution on $\{-1,1\}$ ) distribution. To be more precise, in [13] it is shown that there exists a positive constant $C$ such that for any $t>0$

$$
\limsup _{n \rightarrow \infty} \mathbb{P}\left(\min _{|| z|-1| \leq t n^{-2}}\left|W_{n}(z)\right| \leq t n^{-1 / 2}\right) \leq C t .
$$

Moreover, in (2.3) in [13] the limit

$$
\mathbb{P}\left(\min _{x \in[0,1]}\left|W_{n}(x)\right| \leq n^{-1 / 2}(\log n)^{-\gamma}\right)=\mathrm{o}(1), \quad \text { as } n \rightarrow \infty,
$$

is established for $\gamma>1 / 2$ and iid Gaussian rcs.

Karapetyan in [12] mentioned that it is possible to extend the above result under the assumption of nondegenerate real sub-Gaussian rcs, but only a sketch of the proof was given. Moreover, he claimed that the previous result can be extended under the finiteness of the third moment on the rcs. However, Karapetyan in [11] showed that for iid res with zero mean and finite third moment, it follows, for any $\epsilon \in(0,1)$ and $n>16 C^{\frac{9936}{\epsilon^{3}}}$,

$$
\mathbb{P}\left(\min _{x \in[0,1]}\left|\sum_{j=0}^{n-1} \xi_{j} e^{i j x}\right| \geq n^{-1 / 2+\epsilon}\right) \leq \frac{1}{n^{\epsilon^{2} / 180}},
$$

where the constant $C$ depends only on the moments of $\xi_{0}$. The proof of (5) is long, technical and complicated.

Later, Barrera and Manrique [2] proved that if the moment generating function of iid coefficients exists in an open neighborhood around 0 , then for any $t \geq 1$

$$
\mathbb{P}\left(\min _{|| z|-1| \leq t n^{-2}(\log n)^{-1 / 2-\gamma}}\left|W_{n}(z)\right| \leq t n^{-1 / 2}(\log n)^{-\gamma}\right)=\mathrm{O}\left((\log n)^{-\gamma+1 / 2}\right),
$$

where $\gamma>1 / 2$. The proof of (6) recovers the essential ideas of Konyagin and Schlag [13], who only considered the problem when the rcs have Rademacher or standard Gaussian distribution. Their proof is quite technical and involved. It is based on the Salem-Zygmund inequality for sub-Gaussian rvs.

To extend for more distributions, Barrera and Manrique [2] took advantage of the concept of least common denominator (lcd for short), which was developed in the study of the singularity of random matrices [17]. Roughly speaking, the lcd is a combinatorial measurement to understand the concentration of a sum of independent rvs in a small ball. Furthermore, under the assumptions of the finiteness of the second moment, using similar ideas from Barrera and Manrique [2], it is possible to find an annulus in which $W_{n}$ does not have roots with 
high probability. We remark that the lcd has been converted into a useful tool that allows the analysis of various interesting problems. For instance, it is used in the study of isomorphism between graphs [15] and in the analysis of the condition number for random matrices [18]. In this paper, the lcd is used to understand how small the modulus of a random polynomial near the unit circle can be.

In this work, the lcd allows us to develop clear arguments to estimate how close the roots of a Kac polynomial are to the unit circle. To be more precise, when the rcs of a Kac polynomial are iid rvs with zero mean and finite second moment, the majority of the roots are at a distance of order $\mathrm{O}\left(n^{-2}(\log n)^{-3}\right)$ with probability $1-\mathrm{O}\left((\log n)^{-1 / 2}\right)$. The main obstacle for extending this result comes from the Salem-Zygmund inequality as we will see in Section 2.

The main result of this paper is the following.

Theorem 1.1 Let $\left\{\xi_{j}: j \geq 0\right\}$ be a sequence of real iid nondegenerate real rvs satisfying

$$
\sup _{u \in \mathbb{R}} \mathbb{P}\left(\left|\xi_{0}-u\right| \leq \gamma\right) \leq 1-q \text { and } \mathbb{P}\left(\left|\xi_{0}\right|>M\right) \leq \frac{q}{2}
$$

for some $M>0, \gamma>0$ and $q \in(0,1)$. Suppose $\mathbb{E}\left[\xi_{0}\right]=0$ and $\mathbb{E}\left[\xi_{0}^{2}\right]<\infty$. Then for all fixed $t \geq 1$ we have

$$
\mathbb{P}\left(\min _{|| z|-1| \leq t n^{-2}(\log n)^{-3}}\left|W_{n}(z)\right| \leq t n^{-1 / 2}(\log n)^{-2}\right)=\mathrm{O}\left((\log n)^{-1 / 2}\right),
$$

where the implicit constant depends on $t$ and the distribution of $\xi_{0}$.

Remark 1.2 (1) In Theorem 1.1 we only assume the finiteness of the second moment, zero mean and condition $(\mathbf{H})$ which include Rademacher and standard Gaussian rvs. As a direct consequence of Theorem 1.1, we have

$$
\mathbb{P}\left(W_{n} \text { has no roots on }\left\{z \in \mathbb{C}:|| z|-1| \leq t n^{-2}(\log n)^{-3}\right\}\right)=1-\mathrm{O}\left((\log n)^{-1 / 2}\right) .
$$

(2) We point out that in (7) we consider the minimum of the modulus of the Kac polynomial over the set $\left\{|| z|-1| \leq t n^{-2}(\log n)^{-3}\right\}$ which is properly contained in the region considered in (3), but it contains the region considered in (4). Nevertheless, we obtain the upper bound $\mathrm{O}\left((\log n)^{-1 / 2}\right)$ which improves the bound given in (3).

This paper is organized as follows. In Sect. 2 we give an outline of the proof. In Sect. 3 we provide the proof of Theorem 1.1. Finally, in Appendix A we prove auxiliary results that we used throughout the paper.

\section{Outline of the proof}

In this section, we present the strategy used to prove Theorem 1.1. Our goal is to estimate $\mathbb{P}\left(A_{n}\right)$, where

$$
A_{n}:=\left\{\min _{z \in \mathbb{C}:|| z|-1| \leq t n^{-2}(\log n)^{-3}}\left|W_{n}(z)\right| \leq t n^{-1 / 2}(\log n)^{-2}\right\}
$$

and $t \geq 1$ is a fixed constant. First, motivated by the estimates given in [13, Section 2, p. 4964], we analyze the probability of the events

$$
A_{n, \alpha}:=\left\{\left|W_{n}\left(\exp \left(i 2 \pi x_{\alpha}\right)\right)\right| \leq g_{n}\right\} \text { for } x_{\alpha}=\frac{\alpha}{N_{n}}, \alpha=0, \ldots, N_{n}-1,
$$


where $N_{n}$ and $g_{n}$ are appropriate functions of $n$ (we provide a precise description of them later on). We anticipate that $N_{n} \approx n^{2}(\log (n))^{3}$, which is similar to the number of balls used in [13]. We point out that $N_{n}$ needs to trade off $g_{n}$ in order that the probability of $A_{n, \alpha}$ tends to zero, as $n \rightarrow \infty$.

Second, for each $\alpha=0, \ldots, N_{n}-1$ we analyze the arithmetic structure of the sequence $\left\{\exp \left(i 2 \pi j x_{\alpha}\right): j=0, \ldots, n-1\right\}$ and using the so-called small ball inequalities we prove that $\mathbb{P}\left(A_{n, \alpha}\right) \rightarrow 0$, as $n \rightarrow \infty$. The idea is to apply the Taylor Theorem to approximate $W_{n}$ in small balls with centers at $\exp \left(i 2 \pi x_{\alpha}\right)$. This allows us to write the event $A_{n}$ as the union of events of the form $A_{n, \alpha}$. However, we need to handle the maximum value for the derivative of $W_{n}$ on the unit circle. The latter can be done by a Salem-Zygmund type inequality, which estimates the maximum possible value of a Kac polynomial on the unit circle.

Let $\left\|W_{n}^{\prime}\right\|_{\infty}$ denote the supremum norm of $W_{n}^{\prime}$ over the unit circle. In the case of $\xi_{0}, \ldots, \xi_{n-1}$ being iid sub-Gaussian rvs, a Salem-Zygmund type inequality (in probability) gives

$$
\mathbb{P}\left(\left\|W_{n}\right\|_{\infty}>C_{p} n^{1 / 2}(\log n)^{1 / 2}\right)=\mathrm{O}\left(n^{-2}\right)
$$

for some suitable positive constant $C_{p}$, see for instance [10, Chapter 6, Theorem 2]. In [2], the authors showed that (8) holds for iid zero mean rvs with a finite moment generating function. In this paper, we do not assume the existence of the moment generating function. Instead, we assume the finiteness of the second moment. By applying the majorizing measure method, Weber [21] showed (8) in expectation. To be more precise, let $\xi_{0}, \xi_{1}, \ldots, \xi_{n-1}$ be iid zero mean rvs with finite second moment. Corollary 2 in [21] implies that there exists a positive constant $\tilde{C}$ (that only depends on $\mathbb{E}\left[\xi_{0}^{2}\right]$ ) such that

$$
\mathbb{E}\left[\left\|W_{n}\right\|_{\infty}\right] \leq \tilde{C} n^{1 / 2}(\log n)^{1 / 2} \quad \text { for any } n \in \mathbb{N} .
$$

To improve Theorem 1.1 (using the lcd technique) to more general rcs, we require a refined version of the Salem-Zygmund inequality for rvs without a finite second moment. At the moment, the authors are not able to obtain a Salem-Zygmund type inequality for rvs without the finiteness of the second moment. Later, we apply small ball inequalities to show that

$$
\mathbb{P}\left(\left|W_{n}\left(\exp \left(i 2 \pi x_{\alpha}\right)\right)\right| \leq g_{n}\right) \rightarrow 0, \quad \text { as } n \rightarrow \infty .
$$

Such inequalities allow us to consider more general res and provide a new proof of the main theorem in [13]. To apply small ball inequalities, we analyze the lcd for some specific matrix. In the sequel, we give the definition of the lcd for a matrix. Let $\log _{+} x:=\max \{\log x, 0\}$ for any $x>0$.

Definition 2.1 (Least common denominator (lcd)) Let $L>0$ be a positive number. Let $\|\cdot\|_{2}$ be the standard Euclidean norm and let dist $\left(v, \mathbb{Z}^{M}\right)$ denote the distance between the vector $v \in \mathbb{R}^{M}$ and the set $\mathbb{Z}^{M}$. For a given matrix $V \in \mathbb{R}^{m \times M}$ the lcd is defined as

$$
D(V):=\inf \left\{\|\Theta\|_{2}: \Theta \in \mathbb{R}^{m}, \operatorname{dist}\left(V^{T} \Theta, \mathbb{Z}^{M}\right)<L \sqrt{\log _{+} \frac{\left\|V^{T} \Theta\right\|_{2}}{L}}\right\} .
$$

For a review of the concept of lcd, we recommend [17, Section 7]. For our purposes, in Definition 2.1 we take $m=2, M=n$ and the matrix $V$ is given by

$$
V:=\left[\begin{array}{cccc}
1 & \cos \left(2 \pi x_{\alpha}\right) & \ldots & \cos \left((n-1) 2 \pi x_{\alpha}\right) \\
0 & \sin \left(2 \pi x_{\alpha}\right) & \ldots & \sin \left((n-1) 2 \pi x_{\alpha}\right)
\end{array}\right] .
$$


Set $X=\left[\xi_{0}, \ldots, \xi_{n-1}\right]^{T}$. Observe that

$$
\mathbb{P}\left(\|V X\|_{2} \leq g_{n}\right)=\mathbb{P}\left(\left|W_{n}\left(\exp \left(i 2 \pi x_{\alpha}\right)\right)\right| \leq g_{n}\right) .
$$

Note that if $\operatorname{det}\left(V V^{T}\right)>0$, Theorem 7.5 in [17] implies that for $a>0$ and $t \geq 0$

$$
\mathbb{P}\left(\|a V X\|_{2} \leq t\right) \leq \frac{C^{2} L^{2}}{2 a^{2}\left(\operatorname{det}\left(V V^{T}\right)\right)^{1 / 2}}\left(t+\frac{1}{D(a V)}\right)^{2},
$$

where $L \geq \sqrt{2 / q}$ and the constant $C$ only depends on constants $M, \gamma, q$ specified in Theorem 1.1. By Definition 2.1 it is not hard to deduce that, for any $a>0, D(a V) \geq(1 / a) D(V)$. Recall the inequality $(x+y)^{2} \leq 2 x^{2}+2 y^{2}$ for any $x, y \in \mathbb{R}$. By (9) we deduce

$$
\begin{aligned}
\mathbb{P}\left(\|a V X\|_{2} \leq t\right) & \leq \frac{C^{2} L^{2} t^{2}}{a^{2}\left(\operatorname{det}\left(V V^{T}\right)\right)^{1 / 2}}+\frac{C^{2} L^{2}}{a^{2}\left(\operatorname{det}\left(V V^{T}\right)\right)^{1 / 2}(D(a V))^{2}} \\
& \leq \frac{C^{2} L^{2} t^{2}}{a^{2}\left(\operatorname{det}\left(V V^{T}\right)\right)^{1 / 2}}+\frac{C^{2} L^{2}}{\left(\operatorname{det}\left(V V^{T}\right)\right)^{1 / 2}(D(V))^{2}} .
\end{aligned}
$$

Since $x_{\alpha}=\frac{\alpha}{N_{n}}$, the arithmetic properties of $x_{\alpha}$ given by $\alpha$ and $N_{n}$ should play an important role in the estimates. Depending on the greatest common divisor between $\alpha$ and $N_{n}$, $\operatorname{gcd}\left(\alpha, N_{n}\right)$, we deduce suitable positive lower bounds for $\operatorname{det}\left(V^{T} V\right)$ and $\operatorname{dist}\left(V^{T} \Theta, \mathbb{Z}^{n}\right)$ which together with $(\mathbf{K})$ allow us to show that $\mathbb{P}\left(\|V X\|_{2} \leq g_{n}\right)$ is sufficiently small.

\section{Taylor's approximation}

In the sequel, define the trigonometric random polynomial $T_{n}(x):=\sum_{j=0}^{n-1} \xi_{j} e^{i j x}, x \in \mathbb{R}$, and let $T_{n}^{\prime}$ denote its derivative with respect to $x$. To make the notation shorter, $\Delta_{n}$ denotes the following event:

$$
\Delta_{n}:=\left\{\max _{z \in \mathbb{C}:|| z|-1| \leq 2 t n^{-11 / 10}}\left|W_{n}(z)\right| \leq n^{3 / 2},\left\|T_{n}^{\prime}\right\|_{\infty} \leq C_{0} n^{3 / 2} \log n\right\},
$$

where $C_{0}$ is a positive constant of which we will find the precise value later on. We also let $\mathbb{P}(A, B)$ denote the probability $\mathbb{P}(A \cap B)$ for any two events $A$ and $B$. By the total probability law, we deduce

$$
\begin{aligned}
\mathbb{P}\left(A_{n}\right) \leq & \mathbb{P}\left(A_{n}, \Delta_{n}\right)+\mathbb{P}\left(\left\|T_{n}^{\prime}\right\|_{\infty}>C_{0} n^{\frac{3}{2}} \log n\right) \\
& +\mathbb{P}\left(\max _{z \in \mathbb{C}:|| z|-1| \leq 2 t n^{-11 / 10}}\left|W_{n}(z)\right|>n^{3 / 2}\right) .
\end{aligned}
$$

The Markov inequality yields

$$
\begin{aligned}
& \mathbb{P}\left(\max _{z \in \mathbb{C}:|z|-1 \mid \leq 2 t n^{-11 / 10}}\left|W_{n}(z)\right|>n^{3 / 2}\right) \leq \mathbb{P}\left(\sum_{j=0}^{n-1}\left|\xi_{j}\right|\left(1+\frac{2 t}{n^{1+1 / 10}}\right)^{j}>n^{3 / 2}\right) \\
& \quad \leq \frac{\mathbb{E}\left[\sum_{j=0}^{n-1}\left|\xi_{j}\right|\left(1+\frac{2 t}{n^{1+1 / 10}}\right)^{j}\right]}{n^{3 / 2}} \leq \frac{e^{2 t} n \mathbb{E}\left[\left|\xi_{0}\right|\right]}{n^{3 / 2}}=\frac{e^{2 t} \mathbb{E}\left[\left|\xi_{0}\right|\right]}{n^{1 / 2}}
\end{aligned}
$$

in other words,

$$
\mathbb{P}\left(\max _{z \in \mathbb{C}:|z|-1 \mid \leq 2 t n^{-11 / 10}}\left|W_{n}(z)\right|>n^{3 / 2}\right)=\mathrm{O}\left(n^{-1 / 2}\right),
$$


where the implicit constant depends on $t$ and $\mathbb{E}\left[\left|\xi_{0}\right|\right]$. On the other hand, the Bernstein inequality (see [16, Theorem 14.1.1]) allows us to deduce for the second term on the rightside hand of (10) the inequality

$$
\mathbb{P}\left(\left\|T_{n}^{\prime}\right\|_{\infty}>C_{0} n^{3 / 2} \log n\right) \leq \mathbb{P}\left(\left\|T_{n}\right\|_{\infty}>C_{0} n^{1 / 2} \log n\right) .
$$

Since $\mathbb{E}\left[\xi_{0}\right]=0$ and $\mathbb{E}\left[\xi_{0}^{2}\right]<\infty$, one can apply [21, Corollary 2] which together with the Markov inequality imply

$$
\mathbb{P}\left(\left\|T_{n}\right\|_{\infty}>C_{0} n^{1 / 2} \log n\right) \leq \frac{C\left(\mathbb{E}\left[\xi_{0}^{2}\right]\right)^{1 / 2} n^{1 / 2}(\log n)^{1 / 2}}{C_{0} n^{1 / 2} \log n}=\frac{C\left(\mathbb{E}\left[\xi_{0}^{2}\right]\right)^{1 / 2}}{C_{0}(\log n)^{1 / 2}},
$$

where $C$ is a universal positive constant. Consequently, the Bernstein inequality yields

$$
\mathbb{P}\left(\left\|T_{n}^{\prime}\right\|_{\infty}>C_{0} n^{3 / 2} \log n\right)=\mathrm{O}\left((\log n)^{-1 / 2}\right) .
$$

By (10), (11) and (12), we observe that to estimate $\mathbb{P}\left(A_{n}\right)$ we only need to analyze $\mathbb{P}\left(A_{n}, \Delta_{n}\right)$.

Remark 2.2 In the preceding reasoning we only used zero mean and the finiteness of the second moment of $\xi_{0}$. In particular, it holds for sub-Gaussian rvs, which includes Rademacher, standard Gaussian, and bounded rvs.

\section{Arithmetic properties of $\mathbf{x}$}

In the sequel, we decompose the event $A_{n} \cap \Delta_{n}$ into regions for which the arithmetic properties of $x_{\alpha}$ are useful in allowing the use of the anti-concentration assumption $(\mathbf{H})$ and allowing us to show that $\mathbb{P}\left(A_{n}, \Delta_{n}\right)$ tends to zero, as $n \rightarrow \infty$. We point out that in the following reasoning we use only assumption $(\mathbf{H})$.

To achieve our goal, we consider a set of balls with center at points on the unit circle with an adequate radius. We distinguish two kinds of balls: the special balls with center at $1+0 i$ and $-1+0 i$, where the radius $r$ is large $\left(r=2 t n^{-11 / 10}\right)$, and the balls with center at points $z$ with argument satisfying $n^{-11 / 10}<|\arg (z)|<\pi-n^{-11 / 10}$ and small radius $\left(r=2 t n^{-2}(\log n)^{-3}\right)$.

Recall that for any $x \in \mathbb{R},\lfloor x\rfloor$ denotes the greatest integer less than or equal to $x$. Let $N=\left\lfloor n^{2}(\log n)^{3}\right\rfloor$ and $x_{\alpha}=\frac{\alpha}{N}$ for $\alpha=0,1, \ldots, N-1$. For $a \in \mathbb{C}$ and $s>0$, let B $(a, s)$ denote the closed ball with center $a$ and radius $s$, i.e., $\mathrm{B}(a, s)=\{z \in \mathbb{C}:|z-a| \leq s\}$. Write $\mathbb{S}^{1}$ for the unit circle and let

$$
\mathcal{A}\left(\mathbb{S}^{1}, t n^{-2}(\log n)^{-3}\right):=\left\{z \in \mathbb{C}:|| z|-1| \leq t n^{-2}(\log n)^{-3}\right\} .
$$

Notice that

$$
\begin{aligned}
\mathcal{A}\left(\mathbb{S}^{1}, t n^{-2}(\log n)^{-3}\right)=\{ & \left\{z \in \mathcal{A}: n^{-11 / 10}<|\arg (z)|<\pi-n^{-11 / 10}\right\} \\
& \cup\left\{z \in \mathcal{A}:|\arg (z)| \leq n^{-11 / 10} \text { or }|\arg (z)-\pi| \leq n^{-11 / 10}\right\} .
\end{aligned}
$$

If $t \geq 1$, observe that

$$
\begin{aligned}
& \left\{z \in \mathcal{A}:|\arg (z)| \leq n^{-11 / 10} \text { or }|\arg (z)-\pi| \leq n^{-11 / 10}\right\} \\
& \quad \subset \mathrm{B}\left(-1+0 i, 2 t n^{-11 / 10}\right) \cup \mathrm{B}\left(1+0 i, 2 t n^{-11 / 10}\right) .
\end{aligned}
$$

The preceding inclusion yields that any $z \in \mathcal{A}$ with small argument belongs in the union of the balls with center at $1+0 i$ and $-1+0 i$ with radius $2 t n^{-11 / 10}$. On the other hand, for $z \in \mathcal{A}$ with large argument, 


$$
\begin{aligned}
& \left\{z \in \mathcal{A}: n^{-11 / 10}<|\arg (z)|<\pi-n^{-11 / 10}\right\} \\
& \subset \bigcup_{\substack{\alpha=1 \\
\alpha: n^{-11 / 10}<\left|2 \pi x_{\alpha}\right|<\pi-n^{-11 / 10}}}^{N-1} \mathrm{~B}\left(e^{i 2 \pi x_{\alpha}}, 2 t n^{-2}(\log n)^{-3}\right) .
\end{aligned}
$$

Define

$$
\begin{aligned}
& J_{1}(n, N):=\left\{\alpha \in[1, N-1] \cap \mathbb{N}: \operatorname{gcd}(\alpha, N) \geq n^{11 / 10}(\log n)^{-1 / 2}\right\}, \\
& J_{2}(n, N):=\left\{\alpha \in[1, N-1] \cap \mathbb{N}: n^{11 / 10}(\log n)^{-1 / 2} \geq \operatorname{gcd}(\alpha, N) \geq n(\log n)^{3}\right\}, \\
& J_{3}(n, N):=\left\{\alpha \in[1, N-1] \cap \mathbb{N}: n(\log n)^{3} \geq \operatorname{gcd}(\alpha, N) \geq n^{9 / 10}(\log n)^{3}\right\},
\end{aligned}
$$

where $\operatorname{gcd}(\alpha, N)$ denotes the greatest common divisor of $\alpha$ and $N$.

For any $\alpha \in J_{3}(n, N)$, we have

$$
n-\frac{1}{n(\log n)^{3}} \leq \frac{N}{\operatorname{gcd}(\alpha, N)} \leq n^{11 / 10} .
$$

The preceding inequalities mean that the irreducible fraction of $x_{\alpha}$ is as small as a multiple of $n^{-11 / 10}$. Therefore,

$$
\begin{gathered}
\bigcup_{\substack{\alpha=1 \\
<: n^{-11 / 10}<2 \pi x_{\alpha} \mid<\pi-n^{-11 / 10}}}^{N-1} \mathrm{~B}\left(e^{i 2 \pi x_{\alpha}}, 2 t n^{-2}(\log n)^{-3}\right) \\
=\bigcup_{\alpha \in J_{1}(n, N)} \mathrm{B}\left(e^{i 2 \pi x_{\alpha}}, 2 t n^{-2}(\log n)^{-3}\right) \cup \bigcup_{\alpha \in J_{2}(n, N)} \mathrm{B}\left(e^{i 2 \pi x_{\alpha}}, 2 t n^{-2}(\log n)^{-3}\right) \\
\quad \cup \bigcup_{\alpha \in J_{3}(n, N)} \mathrm{B}\left(e^{i 2 \pi x_{\alpha}}, 2 t n^{-2}(\log n)^{-3}\right) .
\end{gathered}
$$

We emphasize that if $\alpha \in J_{1}(n, N) \cup J_{2}(n, N) \cup J_{3}(n, N)$, then

$$
n^{-11 / 10}<\left|2 \pi x_{\alpha}\right|<\pi-n^{-11 / 10} .
$$

Consequently,

$$
\begin{aligned}
\mathbb{P}\left(A_{n}, \Delta_{n}\right) \leq & \mathbb{P}\left(\Delta_{n}, \min _{z \in \mathrm{B}\left(1+0 i, 2 t n^{-11 / 10}\right)}\left|W_{n}(z)\right|<t n^{-1 / 2}(\log n)^{-2}\right) \\
& +\mathbb{P}\left(\Delta_{n}, \min _{z \in \mathrm{B}\left(-1+0 i, 2 t n^{-11 / 10}\right)}\left|W_{n}(z)\right|<t n^{-1 / 2}(\log n)^{-2}\right) \\
& +\sum_{\alpha \in J_{1}(n, N)} \mathbb{P}\left(\Delta_{n}, \mathrm{~B}_{\alpha}\right)+\sum_{\alpha \in J_{2}(n, N)} \mathbb{P}\left(\Delta_{n}, \mathrm{~B}_{\alpha}\right)+\sum_{\alpha \in J_{3}(n, N)} \mathbb{P}\left(\Delta_{n}, \mathrm{~B}_{\alpha}\right),
\end{aligned}
$$

where

$$
\mathrm{B}_{\alpha}:=\left\{\min _{z \in \mathrm{B}\left(e^{i 2 \pi x_{\alpha}}, 2 t n^{-2}(\log n)^{-3}\right)}\left|W_{n}(z)\right|<t n^{-1 / 2}(\log n)^{-2}\right\} .
$$

The right-hand side of (13) will be estimated as follows. 
Lemma 2.3 The following hold.

$$
\mathbb{P}\left(\Delta_{n}, \min _{z \in \mathrm{B}\left(1+0 i, 2 t n^{-11 / 10}\right)}\left|W_{n}(z)\right|<t n^{-1 / 2}(\log n)^{-2}\right)=\mathrm{O}\left(\frac{\log n}{n^{1 / 10}}\right)
$$

and

$$
\mathbb{P}\left(\Delta_{n}, \min _{z \in \mathrm{B}\left(-1+0 i, 2 t n^{-11 / 10}\right)}\left|W_{n}(z)\right|<t n^{-1 / 2}(\log n)^{-2}\right)=\mathrm{O}\left(\frac{\log n}{n^{1 / 10}}\right),
$$

where the implicit constants in the big $\mathrm{O}$ notation depend on $L$ and $t$.

Lemma 2.4 Suppose $\operatorname{gcd}(\alpha, N) \geq n^{11 / 10}(\log n)^{-1 / 2}$, where $N=\left\lfloor n^{2}(\log n)^{3}\right\rfloor$. Then for a suitable constant $\tilde{C}$ it follows that

$$
\sum_{\alpha \in J_{1}(n, N)} \mathbb{P}\left(\left|W_{n}\left(e^{i 2 \pi x_{\alpha}}\right)\right| \leq \tilde{C} t n^{-1 / 2}(\log n)^{-2}\right)=\mathrm{O}\left(\frac{(\log n)^{4}}{n^{1 / 20}}\right),
$$

where the implicit constant in the big $\mathrm{O}$ notation depends on $L$ and $t$.

Lemma 2.5 Suppose $\frac{n^{11 / 10}}{(\log n)^{1 / 2}} \geq \operatorname{gcd}(\alpha, N) \geq n(\log n)^{3}$, where $N=\left\lfloor n^{2}(\log n)^{3}\right\rfloor$. Then for a suitable constant $\tilde{C}$ it follows that

$$
\sum_{\alpha \in J_{2}(n, N)} \mathbb{P}\left(\left|W_{n}\left(e^{i 2 \pi x_{\alpha}}\right)\right| \leq \tilde{C} t n^{-1 / 2}(\log n)^{-2}\right)=\mathrm{O}\left(\frac{1}{\log n}\right),
$$

where the implicit constant in the big $\mathrm{O}$ notation depends on $L$ and $t$.

Lemma 2.6 Suppose $n(\log n)^{3} \geq \operatorname{gcd}(\alpha, N) \geq n^{9 / 10}(\log n)^{3}$, where $N=\left\lfloor n^{2}(\log n)^{3}\right\rfloor$. Then for a suitable constant $\tilde{C}$ it follows that

$$
\sum_{\alpha \in J_{3}(n, N)} \mathbb{P}\left(\left|W_{n}\left(e^{i 2 \pi x_{\alpha}}\right)\right| \leq \tilde{C} t n^{-1 / 2}(\log n)^{-2}\right)=\mathrm{O}\left(\frac{1}{n^{1 / 10}}\right),
$$

where the implicit constant in the big $\mathrm{O}$ notation depends on $L$ and $t$.

In the sequel, we stress that Theorem 1.1 is just a consequence of what we have already stated up to here. Indeed, combining Lemma 2.3, Lemma 2.4, Lemma 2.5, Lemma 2.6, estimate (11) and estimate (12) in inequality (10) yields Theorem 1.1.

\section{Proof of Theorem 1.1}

In this section, we show that the left-hand side of inequality (13) is of order $\mathrm{O}\left((\log (n))^{-1 / 2}\right)$.

\subsection{Estimates on the balls centered at -1 and 1}

Proof of Lemma 2.3 Let $z \in \mathrm{B}\left(1+0 i, 2 t n^{-11 / 10}\right)$. The Taylor Theorem implies

$$
\left|W_{n}(z)-W_{n}(1)\right| \leq|z-1|\left|W_{n}^{\prime}(1)\right|+\left|R_{2}(z)\right|
$$


where $R_{2}(z)$ is the error of the Taylor approximation of order 2. On $\Delta_{n}$,

$$
\begin{aligned}
\left|R_{2}(z)\right| & \leq \frac{\left(2 t n^{-1-1 / 10}\right)^{2}}{1-\mathrm{o}(1)} \max _{z \in \mathrm{B}\left(1+0 i, 2 t n^{-11 / 10}\right)}\left|W_{n}(z)\right| \\
& \leq \frac{4 t^{2} n^{-2-1 / 5} n^{3 / 2}}{1-\mathrm{o}(1)}=\frac{4 t^{2} n^{-1 / 2-1 / 5}}{1-\mathrm{o}(1)}
\end{aligned}
$$

where $\mathrm{o}(1)=2 t n^{-1-1 / 10}$. By the preceding inequality on $\Delta_{n}$, we infer

$$
\begin{aligned}
\left|W_{n}(z)-W_{n}(1)\right| & \leq 2 t n^{-1-1 / 10}\left|W_{n}^{\prime}(1)\right|+\frac{4 t^{2} n^{-1 / 2-1 / 5}}{1-\mathrm{o}(1)} \\
& \leq 2 t n^{-1-1 / 10}\left\|T_{n}^{\prime}\right\|_{\infty}+\frac{4 t^{2} n^{-1 / 2-1 / 5}}{1-\mathrm{o}(1)} \\
& \leq 2 C_{0} t n^{1 / 2-1 / 10} \log n+\frac{4 t^{2} n^{-1 / 2-1 / 5}}{1-\mathrm{o}(1)} .
\end{aligned}
$$

Hence,

$\mathbb{P}\left(\Delta_{n}, \min _{z \in \mathrm{B}\left(1+0 i, 2 t n^{-11 / 10}\right)}\left|W_{n}(z)\right|<t n^{-1 / 2}(\log n)^{-2}\right) \leq \mathbb{P}\left(\left|W_{n}(1)\right| \leq 2 C_{2} t n^{1 / 2-1 / 10} \log n\right)$,

where $2 C_{2}=2 C_{0}+4 t+1$. Since $W_{n}(1)=\sum_{j=0}^{n-1} \xi_{j}$, [17, Corollary 7.6] implies

$$
\mathbb{P}\left(\left|W_{n}(1)\right| \leq 2 C_{2} t n^{1 / 2-1 / 10} \log n\right) \leq \frac{C_{3} L}{\|\mathbf{a}\|}\left(2 C_{2} t+\frac{1}{D(\mathbf{a})}\right)
$$

for $L \geq \sqrt{1 / q}$, where $C_{3}$ is a positive constant and $D(\mathbf{a})$ is the lcd of

$$
\mathbf{a}=\left(n^{1 / 2-1 / 10} \log n\right)^{-1}(1, \ldots, 1) \in \mathbb{R}^{n} .
$$

By [17, Proposition 7.4], $D(\mathbf{a}) \geq \frac{1}{2|\mathbf{a}|_{\infty}}$, where $|\mathbf{a}|_{\infty}$ denotes the maximum Euclidean norm of the columns of $\mathbf{a}$. Then $D(\mathbf{a}) \geq 1 / 2 n^{1 / 2-1 / 10} \log n$ and

$$
\begin{aligned}
\mathbb{P}\left(\left|W_{n}(1)\right| \leq 2 C_{2} t n^{1 / 2-1 / 10} \log n\right) & \leq \frac{C_{3} L \log n}{n^{1 / 10}}\left(2 C_{2} t+\frac{2}{n^{1 / 2-1 / 10} \log n}\right) \\
& \leq \frac{\left(2 C_{2} t+2\right) C_{3} L \log n}{n^{1 / 10}} .
\end{aligned}
$$

Therefore,

$$
\mathbb{P}\left(\Delta_{n}, \min _{z \in \mathrm{B}\left(1+0 i, 2 t n^{-11 / 10}\right)}\left|W_{n}(z)\right|<t n^{-1 / 2}(\log n)^{-2}\right)=\mathrm{O}\left(\frac{\log n}{n^{1 / 10}}\right) .
$$

On the other hand, for $z \in B\left(-1+0 i, 2 t n^{-11 / 10}\right)$ a similar reasoning yields $\mathbb{P}\left(\Delta_{n}, \min _{z \in \mathrm{B}\left(-1+0 i, 2 t n^{-11 / 10}\right)}\left|W_{n}(z)\right|<t n^{-1 / 2}(\log n)^{-2}\right) \leq \mathbb{P}\left(\left|W_{n}(-1)\right| \leq 2 C_{2} t n^{1 / 2-1 / 10} \log n\right)$.

In this case, we need to analyze $W_{n}(-1)=\sum_{j=0}^{n-1}(-1)^{j} \xi_{j}$. Again taking $L \geq \sqrt{1 / q}$ and applying [17, Corollary 7.6], we obtain

$$
\mathbb{P}\left(\left|W_{n}(-1)\right| \leq 2 C_{2} t n^{1 / 2-1 / 10} \log n\right) \leq \frac{C_{3} L}{\|\mathbf{b}\|}\left(2 C_{2} t+\frac{1}{D(\mathbf{b})}\right),
$$


where $C_{3}$ is a positive constant and $D(\mathbf{b})$ is the lcd of

$$
\mathbf{b}=\left(n^{1 / 2-1 / 10} \log n\right)^{-1}\left(1,-1,1, \ldots,(-1)^{n-1}\right) \in \mathbb{R}^{n} .
$$

By [17, Proposition 7.4], $D(\mathbf{b}) \geq 1 / 2 n^{1 / 2-1 / 10} \log n$. So

$$
\begin{aligned}
\mathbb{P}\left(\left|W_{n}(-1)\right| \leq 2 C_{2} t n^{1 / 2-1 / 10} \log n\right) & \leq \frac{C_{3} L \log n}{n^{1 / 10}}\left(2 C_{2} t+\frac{2}{n^{1 / 2-1 / 10} \log n}\right) \\
& \leq \frac{\left(2 C_{2} t+2\right) C_{3} L \log n}{n^{1 / 10}} .
\end{aligned}
$$

Therefore,

$$
\mathbb{P}\left(\Delta_{n}, \min _{z \in \mathrm{B}\left(-1+0 i, 2 t n^{-11 / 10}\right)}\left|W_{n}(z)\right|<t n^{-1 / 2}(\log n)^{-2}\right)=\mathrm{O}\left(\frac{\log n}{n^{1 / 10}}\right) .
$$

\subsection{Estimates of $\mathbb{P}\left(\Delta_{n}, B_{\alpha}\right)$}

In the sequel, we apply the Taylor Theorem repeatedly in order to reduce $\mathbb{P}\left(\Delta_{n}, \mathrm{~B}_{\alpha}\right)$ to an estimate of the probability of how small a sum of iid rvs can be. The latter can be computed (estimated) using small ball inequalities.

Let $z \in \mathrm{B}\left(e^{i 2 \pi x_{\alpha}}, 2 t n^{-2}(\log n)^{-3}\right)$ and suppose that $\Delta_{n}$ holds. The Taylor Theorem yields

$$
\begin{aligned}
\left|W_{n}(z)-W_{n}\left(e^{i 2 \pi x_{\alpha}}\right)\right| & \leq\left|z-e^{i 2 \pi x_{\alpha}}\right|\left|W_{n}^{\prime}\left(e^{i 2 \pi x_{\alpha}}\right)\right|+\left|R_{2}(z)\right| \\
& \leq 2 t n^{-2}(\log n)^{-3}\left|W_{n}^{\prime}\left(e^{i 2 \pi x_{\alpha}}\right)\right|+\frac{4 t^{2} n^{-5 / 2}(\log n)^{-6}}{1-o(1)} \\
& \leq\left(2 t C_{0}+4 t^{2}\right) n^{-1 / 2}(\log n)^{-2},
\end{aligned}
$$

where o(1) $=2 t n^{-2}(\log n)^{-3}$. Hence

$$
\mathbb{P}\left(\Delta_{n}, \mathrm{~B}_{\alpha}\right) \leq \mathbb{P}\left(\left|W_{n}\left(e^{i 2 \pi x_{\alpha}}\right)\right| \leq 2 t C_{2} n^{-1 / 2}(\log n)^{-2}\right) .
$$

To show that $\mathbb{P}\left(\Delta_{n}, \mathrm{~B}_{\alpha}\right)$ tends to zero as $n$ increases, we rewrite the sum $W_{n}\left(e^{i 2 \pi x_{\alpha}}\right)$ as the product of a matrix and a vector, and then we analyze the lcd of the corresponding matrix.

Define the $2 \times n$ matrix $V_{\alpha}$ as follows:

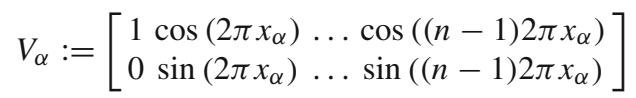

and take $X=\left[\xi_{0}, \ldots, \xi_{n-1}\right]^{T} \in \mathbb{R}^{n}$. Notice that

$$
\left\|V_{\alpha} X\right\|_{2}=\left|\sum_{j=0}^{n-1} \xi_{j} e^{i j 2 \pi x_{\alpha}}\right|=\left|W_{n}\left(e^{i 2 \pi x_{\alpha}}\right)\right| .
$$

Let $\Theta=r[\cos (\theta), \sin (\theta)]^{T} \in \mathbb{R}^{2}$, where $r>0$ and $\theta \in[0,2 \pi)$. For fixed $r$ and $\theta$ we have

$$
V_{\alpha}^{T} \Theta=r\left[\cos (-\theta), \cos \left(2 \pi x_{\alpha}-\theta\right), \ldots, \cos \left(2(n-1) \pi x_{\alpha}-\theta\right)\right]^{T} \in \mathbb{R}^{n} .
$$


We also point out that $\left\|V_{\alpha}^{T} \Theta\right\|_{2} \leq r \sqrt{n}$. On the other hand, we observe that

$$
\operatorname{det}\left(V_{\alpha} V_{\alpha}^{T}\right)=\operatorname{det}\left[\begin{array}{cc}
\sum_{j=0}^{n-1} \cos ^{2}\left(j 2 \pi x_{\alpha}\right) & \frac{1}{2} \sum_{j=0}^{n-1} \sin \left(2 \cdot j 2 \pi x_{\alpha}\right) \\
\frac{1}{2} \sum_{j=0}^{n-1} \sin \left(2 \cdot j 2 \pi x_{\alpha}\right) & \sum_{j=0}^{n-1} \sin ^{2}\left(j 2 \pi x_{\alpha}\right)
\end{array}\right] .
$$

Bearing all this in mind, we can use the notion of lcd for high dimensions to obtain an accurate upper bound of the left-hand side of (14).

We recall that the events $\Delta_{n} \cap \mathrm{B}_{\alpha}$ are defined for

$$
n^{-11 / 10}<\left|2 \pi x_{\alpha}\right|<\pi-n^{-11 / 10} .
$$

Therefore, to estimate the left-hand side of (14), we distinguish the following three cases.

\subsubsection{Estimation on $J_{1}(n, N)$}

Proof of Lemma 2.4 Notice that

$$
\frac{N}{\operatorname{gcd}(\alpha, N)} \leq \frac{n^{2}(\log n)^{3}}{n^{11 / 10}(\log n)^{-1 / 2}}=n^{9 / 10}(\log n)^{7 / 2}
$$

and

$$
\left|2 \pi x_{\alpha}\right|=2 \pi \frac{\alpha}{N}=2 \pi \frac{\alpha / \operatorname{gcd}(\alpha, N)}{N / \operatorname{gcd}(\alpha, N)} \geq 2 \pi \frac{1}{n^{9 / 10}(\log n)^{7 / 2}} .
$$

Then $2 \pi x_{\alpha}$ also satisfies $n^{-1}<\left|2 \pi x_{\alpha}\right|<\pi-n^{-1}$ for all large $n$. By [13, Lemma 3.2, Part 1], there exist positive constants $c_{4}, C_{4}$ such that

$$
c_{4} n^{2} \leq \operatorname{det}\left(V_{\alpha} V_{\alpha}^{T}\right) \leq C_{4} n^{2} .
$$

By Lemma A.1 in Appendix A we obtain that the number of indices $\alpha \in[1, N] \cap \mathbb{N}$ that satisfies the condition $\operatorname{gcd}(\alpha, N) \geq n^{11 / 10}(\log n)^{-1 / 2}$ is at most $\frac{N^{1+o(1)}}{n^{11 / 10}(\log n)^{-1 / 2}}$. By the definition of $N$ we obtain

$$
\frac{N^{1+\mathrm{o}(1)}}{n^{11 / 10}(\log n)^{-1 / 2}} \leq \frac{n^{2+\mathrm{o}(1)}(\log n)^{7 / 2+\mathrm{o}(1)}}{n^{11 / 10}}=n^{9 / 10+\mathrm{o}(1)}(\log n)^{7 / 2+\mathrm{o}(1)} .
$$

By [17, Proposition 7.4], the lcd of $V_{\alpha}$ satisfies $D\left(V_{\alpha}\right) \geq \frac{1}{2\left|V_{\alpha}\right|_{\infty}}$, where $\left|V_{\alpha}\right|_{\infty}$ denotes the maximum Euclidean norm of the columns of $V_{\alpha}$. Observe that $\left|V_{\alpha}\right|_{\infty}=1$ and hence $D\left(V_{\alpha}\right) \geq 1 / 2$. Therefore, inequality (K), inequality (15) and inequality (16) yield

$$
\begin{aligned}
\sum_{\alpha \in J_{1}(n, N)} \mathbb{P} & \left(\left|W_{n}\left(e^{i 2 \pi x_{\alpha}}\right)\right| \leq 2 t C_{2} n^{-1 / 2}(\log n)^{-2}\right) \\
& \leq n^{9 / 10+o(1)}(\log n)^{7 / 2+o(1)}\left(\frac{2 C^{2} L^{2}\left(2 t C_{2}\right)^{2}}{\left(c_{4} n^{2}\right)^{1 / 2}\left(n^{1 / 2}(\log n)^{2}\right)^{2}}+\frac{2 C^{2} L^{2}}{\frac{1}{4}\left(c_{4} n^{2}\right)^{1 / 2}}\right) \\
& \leq \frac{8 C^{2} C_{2}^{2} L^{2} t^{2}}{c_{4}^{1 / 2} n^{11 / 10-o(1)}(\log n)^{1 / 2-o(1)}}+\frac{8 C^{2} L^{2}(\log n)^{7 / 2+o(1)}}{c_{4}^{1 / 2} n^{1 / 10-o(1)}}
\end{aligned}
$$

for all large $n$. Consequently,

$$
\sum_{\alpha \in J_{1}(n, N)} \mathbb{P}\left(\left|W_{n}\left(e^{i 2 \pi x_{\alpha}}\right)\right| \leq 2 t C_{2} n^{-1 / 2}(\log n)^{-2}\right)=\mathrm{O}\left(\frac{(\log n)^{4}}{n^{1 / 20}}\right),
$$


where the implicit constant depends on $L$ and $t$.

\subsubsection{Estimation on $J_{2}(n, N)$}

Proof of Lemma 2.5 Notice that

$$
n \geq \frac{N}{\operatorname{gcd}(\alpha, N)} \geq n^{9 / 10}(\log n)^{7 / 2}-\mathrm{o}(1),
$$

where $\mathrm{o}(1)=\frac{(\log n)^{1 / 2}}{n^{11 / 10}}$. The latter implies

$$
\left|2 \pi x_{\alpha}\right|=2 \pi \frac{\alpha}{N}=2 \pi \frac{\alpha / \operatorname{gcd}(\alpha, N)}{N / \operatorname{gcd}(\alpha, N)} \geq 2 \pi \frac{1}{n} .
$$

Then $2 \pi x_{\alpha}$ also satisfies $n^{-1} \leq\left|2 \pi x_{\alpha}\right| \leq \pi-n^{-1}$ for all large $n$. By [13, Lemma 3.2, Part 1] there exist positive constants $c_{4}, C_{4}$ such that

$$
c_{4} n^{2} \leq \operatorname{det}\left(V_{\alpha} V_{\alpha}^{T}\right) \leq C_{4} n^{2}
$$

Note $x_{\alpha}=\frac{\alpha}{N}=\frac{\alpha^{\prime}}{N^{\prime}}$, where $\alpha=\alpha^{\prime} \operatorname{gcd}(\alpha, N)$ and $N=N^{\prime} \operatorname{gcd}(\alpha, N)$. Observe that $\operatorname{gcd}\left(\alpha^{\prime}, N^{\prime}\right)=1$. Since $N^{\prime} \leq n$, for any $\theta$ we have

$$
\left\{\exp \left(i\left(j 2 \pi \frac{\alpha^{\prime}}{N^{\prime}}-\theta\right)\right): j=0, \ldots, N^{\prime}-1\right\}=\left\{\exp \left(i\left(j 2 \pi \frac{1}{N^{\prime}}-\theta\right)\right): j=0, \ldots, N^{\prime}-1\right\}
$$

Hence, without loss of generality, we assume that $x_{\alpha}=\frac{1}{N^{\prime}}$. A straightforward computation yields

$$
V_{\alpha}^{T} \Theta=r\left[\cos (-\theta), \cos \left(2 \pi x_{\alpha}-\theta\right), \ldots, \cos \left(2(n-1) \pi x_{\alpha}-\theta\right)\right]^{T} \in \mathbb{R}^{n}
$$

Notice that the proof of Lemma A.2 in Appendix A holds true for any real positive number $r$. If $r \leq \frac{1}{32 \pi x_{\alpha}}$, by Lemma A.2 in Appendix A, inequality (17) and remembering that $\left\|V_{\alpha}^{T} \Theta\right\|_{2} \leq r \sqrt{n}$ we deduce

$$
\begin{gathered}
\frac{1}{128 \pi}\left(n^{9 / 10}(\log n)^{7 / 2}-\mathrm{o}(1)\right) \leq \frac{1}{128 \pi x_{\alpha}} \leq \operatorname{dist}\left(V_{\alpha}^{T} \Theta, \mathbb{Z}^{n}\right) \\
\leq L \sqrt{\log _{+} \frac{\left\|V_{\alpha}^{T} \Theta\right\|_{2}}{L}} \leq L \sqrt{\log _{+} \frac{r n^{1 / 2}}{L}} \leq L \sqrt{\log _{+} \frac{n^{3 / 2}}{L}},
\end{gathered}
$$

which yields a contradiction as $L \geq \sqrt{2 / q}$ is fixed. Then for $r>\frac{1}{32 \pi x_{\alpha}}$ we have

$$
D\left(V_{\alpha}\right) \geq r>\frac{1}{32 \pi}\left(n^{9 / 10}(\log n)^{7 / 2}-o(1)\right) .
$$


Therefore, the preceding inequality together with inequality (K), inequality (18) and the fact that the cardinality of $J_{2}(n, N)$ is at most $N$ allow us to deduce

$$
\begin{aligned}
& \sum_{\alpha \in J_{2}(n, N)} \mathbb{P}\left(\left|W_{n}\left(e^{i 2 \pi x_{\alpha}}\right)\right| \leq 2 t C_{2} n^{-1 / 2}(\log n)^{-2}\right) \\
& \leq n^{2}(\log n)^{3}\left(\frac{2 C^{2} L^{2}\left(2 t C_{2}\right)^{2}}{\left(c_{4} n^{2}\right)^{1 / 2}\left(n^{1 / 2}(\log n)^{2}\right)^{2}}\right) \\
&+n^{2}(\log n)^{3}\left(\frac{2 C^{2} L^{2}}{\left(c_{4} n^{2}\right)^{1 / 2}\left(\frac{1}{32 \pi}\left(n^{9 / 10}(\log n)^{7 / 2}-o(1)\right)\right)^{2}}\right) \\
& \leq \frac{8 C^{2} C_{2}^{2} L^{2} t^{2}}{c_{4}^{1 / 2} \log n}+\frac{2048 c \pi^{2} C^{2} L^{2}}{c_{4}^{1 / 2} n^{2 / 5}(\log n)^{4}}
\end{aligned}
$$

for all large $n$, where $c_{4}$ is a positive constant. As a consequence we obtain

$$
\sum_{\alpha \in J_{2}(n, N)} \mathbb{P}\left(\left|W_{n}\left(e^{i 2 \pi x_{\alpha}}\right)\right| \leq 2 t C_{2} n^{-1 / 2}(\log n)^{-2}\right)=\mathrm{O}\left(\frac{1}{\log n}\right),
$$

where the implicit constant depends on $L$ and $t$.

\subsubsection{Estimation on $J_{3}(n, N)$}

Proof of Lemma 2.6 This case requires a more refined analysis. Observe that

$$
n^{11 / 10} \geq \frac{N}{\operatorname{gcd}(\alpha, N)} \geq n-\mathrm{o}(1),
$$

where o $(1)=\frac{1}{n^{2}(\log n)^{3}}$. Then $2 \pi x_{\alpha}$ satisfies

$$
n^{-11 / 10} \leq\left|2 \pi x_{\alpha}\right| \leq(n-\mathrm{o}(1))^{-1} \quad \text { or } \quad \pi-(n-\mathrm{o}(1))^{-1} \leq\left|2 \pi x_{\alpha}\right| \leq \pi-n^{-11 / 10} .
$$

By [13, Lemma 3.2, Part 2], there exist positive constants $c_{4}, C_{4}$ such that

$$
c_{4} n^{2-1 / 5} \leq \operatorname{det}\left(V_{\alpha} V_{\alpha}^{T}\right) \leq C_{4} n^{2} .
$$

By Lemma A.1, the number of indexes $\alpha \in[1, N] \cap \mathbb{N}$ that satisfy the condition $n(\log n)^{3} \geq$ $\operatorname{gcd}(\alpha, N) \geq n^{9 / 10}(\log n)^{3}$ is at most $n^{11 / 10+\mathrm{o}(1)}(\log (n))^{\mathrm{o}(1)}$, where $\mathrm{o}(1) \rightarrow 0$, as $n \rightarrow \infty$.

In the sequel, we analyze the lcd of $V_{\alpha}$. In particular, we find an appropriate lower bound for the distance between $V_{\alpha}^{T} \Theta$ and the set $\mathbb{Z}^{n}$. Since $x_{\alpha}=\frac{\alpha}{N}=\frac{\alpha^{\prime}}{N^{\prime}}$ with $\operatorname{gcd}\left(\alpha^{\prime}, N^{\prime}\right)=1$ and $N^{\prime} \geq n-1$ for all large $n$, then we have that all the points in $\left\{\exp \left(i\left(j 2 \pi x_{\alpha}-\theta\right)\right): j=0, \ldots, n-1\right\}$ are. Let $r \in \mathbb{N}$ and consider the set of intervals of the form $\left[\frac{m}{r}, \frac{m+1}{r}\right]$ for all $m \in[-r, r-1] \cap \mathbb{Z}$. Write $I_{m}^{r}$ and $J_{m}^{r}$ for the corresponding arcs on the unit circle such that their projections on the horizontal axis belong to the interval $\left[\frac{m}{r}, \frac{m+1}{r}\right]$. If $4 r \leq n$, then the Pigeonhole Principle implies that there exists at least one $M \in[-r, r-1] \cap \mathbb{Z}$ such that $I_{M}$ or $J_{M}$ contains at least $\frac{n}{4 r} \geq 1$ elements of the set $\left\{\exp \left(i\left(j 2 \pi x_{\alpha}-\theta\right)\right): j=0, \ldots, n-1\right\}$.

In the sequel, we write

$$
I_{M}^{r}:=\left\{j \in\{0, \ldots, n-1\}: \cos (j 2 \pi x-\theta) \in\left[\frac{M}{r}, \frac{M+1}{r}\right] \in\left[\frac{M}{r}, \frac{M+1}{r}\right]\right\} \neq \emptyset,
$$


and, for each $j \in I_{M}^{r}$, we define

$$
d_{j}=\min \left\{\left|\cos \left(j 2 \pi x_{\alpha}-\theta\right)-\frac{M}{r}\right|,\left|\cos \left(j 2 \pi x_{\alpha}-\theta\right)-\frac{M+1}{r}\right|\right\} .
$$

Note that

$$
\min _{0 \leq l<k \leq n-1}\left|l 2 \pi x_{\alpha}-k 2 \pi x_{\alpha}\right| \geq \frac{2 \pi \alpha^{\prime}}{N^{\prime}} \geq \frac{2 \pi}{N^{\prime}} .
$$

Let $L=\min \left\{\left\lfloor\frac{n}{8 r}-\frac{3}{2}\right\rfloor,\left\lfloor\frac{N^{\prime}}{8 r}-\frac{1}{2}\right\rfloor\right\}$ and observe that for each $0 \leq \lambda \leq L$ there exists at least $j \in I_{M}^{r}$ such that $d_{j} \geq(2 \lambda+1) \frac{2 \pi}{N^{\prime}}$. Then

$$
s_{M}^{r}:=\sum_{j \in I_{M}^{r}} d_{j} \geq \sum_{\lambda=0}^{L}(2 \lambda+1) \frac{2 \pi}{N^{\prime}}=\frac{2 \pi(L+1)^{2}}{N^{\prime}} \geq \frac{2 \pi L^{2}}{N^{\prime}} .
$$

By the choice of $L$, if $r \leq\left\lfloor n^{1 / 4}\right\rfloor$, then $\frac{2 \pi L^{2}}{N^{\prime}} \geq \frac{2 \pi}{n^{11 / 10}} n^{3 / 2}$ for all large $n$.

Here, let $v$ be a vector in $\mathbb{R}^{n}$ with entries $v_{j}=\cos \left(j 2 \pi x_{\alpha}-\theta\right)$ for each $j=0, \ldots, n-$ 1. If $r$ is a positive integer with $r \leq\left\lfloor n^{1 / 4}\right\rfloor$, then by the previous discussion we deduce $\operatorname{dist}\left(r v, \mathbb{Z}^{n}\right) \geq 2 \pi n^{2 / 5}$ for all large $n$. If $r$ is any positive real number, observe that $\left[\frac{s}{r}, \frac{s+1}{r}\right] \subset$ $\left[\frac{s}{\lceil r\rceil}, \frac{s+1}{\lceil r\rceil}\right]$, where $s \in \mathbb{N}$, and therefore our previous analysis holds true for any $r>0$.

Suppose $r \leq\left\lfloor n^{1 / 4}\right\rfloor$ and recall that $\left\|V_{\alpha}^{T} \Theta\right\|_{2} \leq r \sqrt{n}$ and that $L \geq \sqrt{2 / q}$ is fixed. By the definition of lcd, for all large $n$, we obtain

$$
2 \pi n^{2 / 5} \leq \operatorname{dist}\left(V_{\alpha}^{T} \Theta, \mathbb{Z}^{n}\right) \leq L \sqrt{\log _{+} \frac{\left\|V_{\alpha}^{T} \Theta_{2}\right\|_{2}}{L}} \leq L \sqrt{\log _{+} \frac{n^{3 / 4}}{L}},
$$

which yields a contradiction for $n$ large. Thus, $D\left(V_{\alpha}\right) \geq\left\lfloor n^{1 / 4}\right\rfloor$. Therefore, the preceding inequality together with inequality $(\mathbf{K})$, inequality (19) and the fact that the cardinality of $J_{3}(n, N)$ is at most $n^{11 / 10+o(1)}(\log (n))^{\mathrm{o}(1)}$ allow us to deduce

$$
\begin{aligned}
& \sum_{\alpha \in J_{3}(n, N)} \mathbb{P}\left(\left|W_{n}\left(e^{i 2 \pi x_{\alpha}}\right)\right| \leq 2 t C_{2} n^{-1 / 2}(\log n)^{-2}\right) \\
\leq & n^{11 / 10+\mathrm{o}(1)}(\log (n))^{\mathrm{o}(1)}\left(\frac{2 C^{2} L^{2}\left(2 t C_{2}\right)^{2}}{\left(c_{4} n^{2-1 / 5}\right)^{1 / 2}\left(n^{1 / 2}(\log n)^{2}\right)^{2}}\right) \\
& +n^{11 / 10+\mathrm{o}(1)}(\log (n))^{\mathrm{o}(1)}\left(\frac{2 C^{2} L^{2}}{\left(c_{4} n^{2-1 / 5}\right)^{1 / 2}\left(n^{1 / 4}\right)^{2}}\right) \\
\leq & \frac{8 C^{2} C_{2}^{2} L^{2} t^{2}}{c_{4}^{1 / 2} n^{4 / 10}}+\frac{2 C^{2} L^{2}}{c_{4}^{1 / 2} n^{1 / 10}}
\end{aligned}
$$

for all large $n$. As a consequence we obtain

$$
\sum_{\alpha \in J_{3}(n, N)} \mathbb{P}\left(\left|W_{n}\left(e^{i 2 \pi x_{\alpha}}\right)\right| \leq 2 t C_{2} n^{-1 / 2}(\log n)^{-2}\right)=\mathrm{O}\left(\frac{1}{n^{1 / 10}}\right),
$$

where the implicit constant depends on $L$ and $t$. 
Acknowledgements Both authors are grateful to Professor Jesús López Estrada for his reading and recommendations in the preliminary version of this work. G. Barrera acknowledges support from a post-doctorate grant held at the Center for Research in Mathematics, (CIMAT, 2015-2016). He would like to express his gratitude to the Pacific Institute for the Mathematical Sciences (PIMS, 2017-2019) for the grant held at the Department of Mathematical and Statistical Sciences at University of Alberta. He also would like to thank CIMAT and the University of Alberta for all the facilities used in the realization of this paper. P. Manrique acknowledges support from Cátedras CONACYT for the research position held at the Mathematics Institute, Cuernavaca (UNAM, 2017-2020). He would also like to thank UNAM for all the facilities used in the realization of this paper. Both authors would like to thank the constructive and useful suggestions provided by the anonymous referee.

Funding Open access funding provided by University of Helsinki including Helsinki University Central Hospital.

Open Access This article is licensed under a Creative Commons Attribution 4.0 International License, which permits use, sharing, adaptation, distribution and reproduction in any medium or format, as long as you give appropriate credit to the original author(s) and the source, provide a link to the Creative Commons licence, and indicate if changes were made. The images or other third party material in this article are included in the article's Creative Commons licence, unless indicated otherwise in a credit line to the material. If material is not included in the article's Creative Commons licence and your intended use is not permitted by statutory regulation or exceeds the permitted use, you will need to obtain permission directly from the copyright holder. To view a copy of this licence, visit http://creativecommons.org/licenses/by/4.0/.

\section{Appendix A: Arithmetic properties}

This section contains the proofs of the results that we skipped in the paper in order to be more fluid.

Lemma A.1 If $m \geq 1$ and $M \in \mathbb{N}$, then the cardinality of the set

$$
\Gamma_{m}^{M}:=\{k \in[1, M] \cap \mathbb{N}: \operatorname{gcd}(k, M) \geq m\}
$$

is at most $\frac{1}{\lfloor m\rfloor} M^{1+C(\log \log M)^{-1}}$, where $C$ is a positive constant.

Proof Let $T$ denote the Euler totient function. Observe that

$$
\sum_{k \in \Gamma_{m}^{M}} 1 \leq \sum_{\substack{d=\lfloor m\rfloor \\ d \mid M}}^{M} T\left(\frac{M}{d}\right)
$$

It is well known that $T(s) \leq s-\sqrt{s}$ for all $s \in \mathbb{N}$. Moreover, if $d(s)$ denotes the number of positive divisors of $s$, then [1, Theorem 13.12] implies that there exists a positive constant $C$ such that $d(s) \leq s^{C(\log \log (s))^{-1}}$. Hence,

$$
\sum_{k \in \Gamma_{m}^{M}} 1 \leq\left(\frac{M}{\lfloor m\rfloor}-\sqrt{\frac{M}{\lfloor m\rfloor}}\right) M^{C(\log \log (M))^{-1}} \leq \frac{1}{\lfloor m\rfloor} M^{1+C(\log \log M)^{-1}}
$$

which yields the statement.

Lemma A.2 Let $\theta \in[0,2 \pi)$ and $n \in \mathbb{N}$. Let $\mathcal{V}=\left(\mathcal{V}_{j}\right)_{j \in\{1, \ldots, n\}} \in \mathbb{R}^{n}$ such that $\mathcal{V}_{j}=$ $r \cos (j 2 \pi x-\theta)$ for $j=0, \ldots, n-1$, where $r \in \mathbb{N}$ and $x=1 / n$. If $\frac{1}{4 \pi r x} \geq 8$, then

$$
\operatorname{dist}\left(\mathcal{V}, \mathbb{Z}^{n}\right) \geq \frac{1}{128 \pi x} \text {. }
$$


Proof Let $\theta \in[0,2 \pi)$ and $n \in \mathbb{N}$. Let $x=1 / n$ and we define the following sequence: $P_{n}=\{\exp (i(j 2 \pi x-\theta)): j=0, \ldots, n-1\}$, where $i$ is the imaginary unit. Note that $P_{n}$ is a set of points on the unit circle which can be viewed as vertices of a regular polygon with $n$ sides inscribed in the unit circle.

Since the arguments of "two consecutive points" on $P_{n}, \exp (i(j 2 \pi x-\theta))$ and $\exp (i((j+1) 2 \pi x-\theta))$, are separated by a distance $2 \pi x$, the number of points in $P_{n}$ which are in any arc of length $\ell$ on the unit circle is at least $\frac{\ell}{2 \pi x}-2$.

Let $[y, y+8 \pi x]$ be a subinterval of $[-1,1]$. We consider an arc $\widehat{I}$ on the unit circle such that its projection on the horizontal axis is $[y, y+8 \pi x]$. If the length of the arc $\widehat{I}$ is $\ell$, then the number of values $\cos (j 2 \pi x-\theta), j=0, \ldots, n-1$, that belong to $(y, y+8 \pi x)$ is at least $\frac{1}{2}\left(\frac{\ell}{2 \pi x}-2\right)$. Observe that $\frac{1}{2}\left(\frac{\ell}{2 \pi x}-2\right) \geq 1$ when $\ell \geq 8 \pi x$.

Let $r \in \mathbb{N}$ and $m \in[-(r-1),(r-1)] \cap \mathbb{Z}$. By the preceding explanation, for all positive integers $k \leq \frac{1}{8 \pi r x}$, there exists $j \in\{0, \ldots, n-1\}$ such that

$$
\cos (j 2 \pi x-\theta) \in\left(\frac{m}{r}+8 \pi(k-1) x, \frac{m}{r}+8 \pi k x\right) \subset\left[\frac{m}{r}, \frac{m+1}{r}\right] .
$$

In the sequel, set

$$
I_{m}^{r}:=\left\{j \in\{0, \ldots, n-1\}: \cos (j 2 \pi x-\theta) \in\left[\frac{m}{r}, \frac{m+1}{r}\right]\right\} \neq \emptyset
$$

and, for each $j \in I_{m}^{r}$, define

$$
d_{j}:=\min \left\{\left|\cos (j 2 \pi x-\theta)-\frac{m}{r}\right|,\left|\cos (j 2 \pi x-\theta)-\frac{m+1}{r}\right|\right\} .
$$

Let $L$ be the biggest integer such that $8 \pi L x \leq \frac{1}{2 r}$, or, equivalently, $L=\left\lfloor\frac{1}{16 \pi r x}\right\rfloor$. Observe that

$$
L \geq \frac{1}{16 \pi r x}-1 \geq \frac{1}{32 \pi r x} \text { when } \quad \frac{1}{4 \pi r x} \geq 8
$$

Then

$$
s_{m}^{r}:=\sum_{j \in I_{m}^{r}} d_{j} \geq \sum_{\lambda=1}^{L} 2 \lambda(8 \pi x) \geq 8 \pi x L^{2} \geq \frac{1}{128 \pi r^{2} x} .
$$

Moreover,

$$
\sum_{m=-(r-1)}^{m=r-1} s_{m}^{r} \geq \frac{2 r-1}{128 \pi r^{2} x} \geq \frac{1}{128 \pi r x}
$$

where the last inequality follows since $\frac{2 r-1}{r} \geq 1$ for $r \in \mathbb{N}$. Consequently, the distance between the vector $\mathcal{V} \in \mathbb{R}^{n}$ with entries $\mathcal{V}_{j}=r \cos (j 2 \pi x-\theta)$ for $j=0, \ldots, n-1$ with $x=1 / n$ and the set $\mathbb{Z}^{n}$ is at least

$$
r\left(\frac{1}{128 \pi r x}\right)=\frac{1}{128 \pi x} \text { verifying that } \quad \frac{1}{4 \pi r x} \geq 8 \text { is fulfilled. }
$$




\section{References}

1. T. Apostol, Introduction to analytic number theory. Undergraduate Texts in Mathematics, Springer-Verlag, (1976)

2. G. Barrera, P. Manrique, Salem-Zygmund inequality for locally sub-Gaussian random variables, random trigonometric polynomials, and random circulant matrices. Submitted. arXiv:1610.05589

3. E. Beller, D. Newman, The minimum modulus of polynomials. Proc. Am. Math. Soc. 45-3, 463-465 (1974)

4. B. Bozkurt, Zeros of the $z$-transform (ZZT) representation and chirp group delay processing for the analysis of source and filter characteristics of speech signals. Ph.d. thesis (2005). https://theses.eurasip. $\mathrm{org} / \mathrm{media} /$ theses/documents/bozkurt-baris-zeros-of-the-z-transform-zzt-representation-and-chirpgroup-delay-processing-for-the-analysis-of-source-and-filter-characteristics-of-speech-signals.pdf . Retrieved 2020 March 20

5. T. Drugman, Y. Stylianou, Fast and accurate phase unwrapping. Proceedings of the INTERSPEECH 2015 : 16th Annual Conference of the International Speech Communication Association, 1171-1175, (2015)

6. F. Götze, D. Zaporozhets, On the distribution of complex roots of random polynomials with heavy-tailed coefficients. Theory Probab. Appl. 4-56, 696-703 (2012)

7. I. Ibragimov, D. Zaporozhets, On distribution of zeros of random polynomials in complex plane. Prokhorov and Contemporary Probability Theory, Proceedings in Mathematics and Statistics 33, Editors: A. Shiryaev, S. Varadhan \& E. Presman. Springer, 303-323 (2013)

8. I. Ibragimov, O. Zeitouni, On the roots of random polynomials. Trans. Am. Math. Soc. 349-6, 2427-2441 (1997)

9. Z. Kabluchko, D. Zaporozhets, Asymptotic distribution of complex zeros of random analytic functions. Ann. Probab. 42-4, 1374-1395 (2014)

10. J. Kahane, Some Random Series of Functions, 2nd edn. (Cambridge University Press, 1985)

11. A. Karapetyan, An estimate of the minimum of the absolute value of trigonometric polynomials with random coefficients. Fundam. Prikl. Mat. 4-1, 245-302 (1998)

12. A. Karapetyan, The values of stochastic polynomials in a neighborhood of the unit circle. Math. Notes 63-1, 127-130 (1998)

13. S. Konyagin, W. Schlag, Lower bounds for the absolute value of random polynomials on a neighbourhood of the unit circle. Trans. Am. Math. Soc. 351-12, 4963-4980 (1999)

14. V. Livshyts, K. Tikhomirov, R. Vershynin, The smallest singular value of inhomogeneous square random matrices. To appear Ann. Probab. 49-3, 1286-1309 (2021)

15. K. Luh, V. Vu, Sparse random matrices have simple spectrum. Ann. Inst. Henri Poincaré Probab. Stat. 56-4, 2307-2328 (2020)

16. Q. Rahman, G. Schmeisser, Analytic theory of polynomials: critical points, zeros and extremal properties. Oxford Science Publications (2002)

17. M. Rudelson, R. Vershynin, No-gaps delocalization for general random matrices. Geom. Funct. Anal. 26-6, 1716-1776 (2016)

18. M. Rudelson, R. Vershynin, The Littlewood-Offord problem and invertibility of random matrices. Adv. Math. 218-2, 600-633 (2008)

19. L. Shepp, R. Vanderbei, The complex zeros of random polynomials. Trans. Am. Math. Soc. 347-11, 4365-4384 (1995)

20. D. Sparo, M. Sur, On the distribution of roots of random polynomials. Vestnik Moskov. Univ. Ser. I Mat. Meh. 3, 40-43 (1962)

21. M. Weber, On a stronger form of Salem-Zygmunds inequality for random trigonometric sums with examples. Period. Math. Hungar. 52-2, 73-104 (2006)

Publisher's Note Springer Nature remains neutral with regard to jurisdictional claims in published maps and institutional affiliations. 